\title{
Assessment of patient-specific efficacy of chemo- and targeted- therapies: a micropharmacology approach
}

\author{
Aleksandra Karolak-Vanderbilt PSOP Member, Branton Huffstutler, Zain Khan \\ and Katarzyna A. Rejniak-Vanderbilt PSOP Member
}

\begin{abstract}
Both targeted and standard chemotherapy drugs are subject to various intratumoral barriers that impede their effectiveness. The tortuous vasculature, dense and fibrous extracellular matrix, irregular cellular architecture, and nonuniform expression of cell membrane receptors hinder drug molecule transport and perturb its cellular uptake. In addition, tumor microenvironments undergo dynamic spatio-temporal changes during tumor progression and treatment, which can also obstruct drug efficacy. To examine these aspects of drug delivery on a cell-to-tissue scale (single-cell pharmacology), we developed the microPKPD models and coupled them with patient-specific data to test personalized treatments.
\end{abstract}

\section{INTRODUCTION TO SINGLE-CELL CANCER PHARMACOLOGY}

The clinical success or failure of targeted and chemotherapy treatments depends on how well a drug's molecules reach all tumor cells (pharmacokinetics, PK) and engage with their molecular targets to invoke the desired therapeutic effect (pharmacodynamics, PD). Conventional PK/PD analyses assess treatment efficacy of the entire organ level, however how drugs penetrate in vivo tissues and how they interact with tumor cells are still poorly understood. Recent advancements in imaging techniques have given rise to a new research area of in vivo single-cell pharmacology [1-3]. The microPKPD (micro-scale PK/PD) modeling framework brings additional power for high throughput simultaneous examination of various physicochemical properties of both drugs and tissues, which provides a tool for improving both drug design and administration protocols that aim to increase anti-cancer treatment efficacy.

\section{ILLUSTRATIVE RESULTS OF MICROPKPD APPLICATIONS}

The crucial advantage of the microPKPD model is the use of digitized tumor tissues as the model domain. This allows for the testing of the treatments that are calibrated to a specific tissue structure, a particular membrane receptor expression, and precise drug-tumor cell interactions. Here, we discuss the application of microPKPD to both biological and medical data on different scales, including patients' biopsies, tumor organoids, and tumor tissue slices.

Research supported by NIH Physical Sciences Oncology Network Grant U01CA202229-02.

A. Karolak, B. Huffstutler, Z. Khan and K.A. Rejniak are with the Integrated Mathematical Oncology Department of the H. Lee Moffitt Cancer Center \& Research Institute, Tampa FL 33612, USA (e-mails: Aleksandra.Karolak@moffitt.org, Kasia.Rejniak@moffitt.org).

\section{A. Predicting a patient's tumor chemoresistance with the Virtual Clinical Trials concept}

The Virtual Clinical Trials concept [4] uses standard-of-care (SOC) medical histology images that are routinely collected in the clinic, advanced image analysis algorithms, and computational simulations to determine whether a patient's tumor is resistant to SOC treatments. We envision that the patient will follow the schematics presented in Figure 1. Tissue samples will be collected from a routine biopsy procedure (A). Following current clinical practices, the tissue will be stained with hematoxylin and eosin (H\&E), sliced, fixed on a glass slide (B), and examined by a pathologist (C). For virtual trials analysis, it will also be scanned and digitized (D). The magnified, high-resolution images will be used to identify and quantify the immunohistochemical (IHC) and morphological features of individual cells (D2, Pathomics). These will be used to characterize the tumor's metabolic landscape pathology $[5,6]$ and to define cellular phenotypes (E, Virtual Pathology). The patient's digitized tissue slices (the z-stack) will be used to reconstruct the entire $3 \mathrm{D}$ tumor organoid (vasculature and cellularity), and the microPKPD model will simulate the $\operatorname{drug}(\mathrm{s})$ penetration and tumor response curves (E2). The likelihood of the tumor being either responsive or resistant to the therapy will be calculated to support clinical decisions (F). In addition, the virtual clinical trials model can be used to predict optimal drug administration protocols (the order, dosage, and timing of a combination of drugs) for personalized treatment.

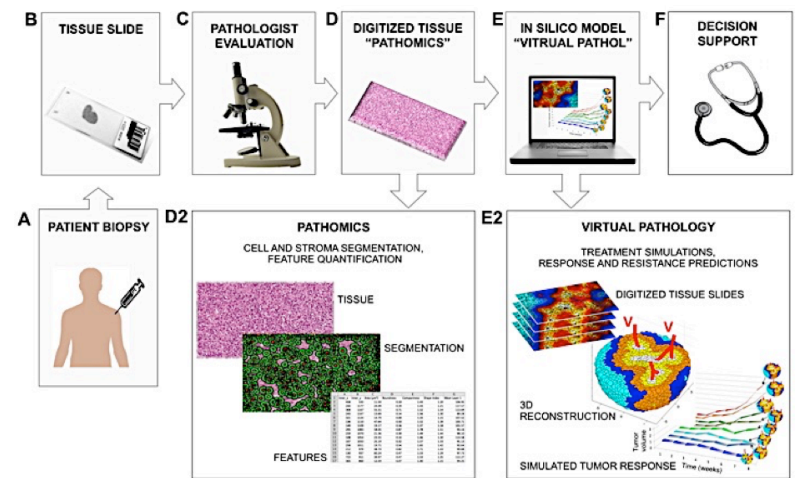

Figure 1. Virtual Clinical Trial "VIRTUOSO" concept for predicting tumor response to chemotherapy based on patients' biopsy data, quantitative pathology analysis and mathematical modeling [4]. 


\section{B. Predicting efficacy of targeted therapy with microPKPD}

Targeted therapies are designed to decrease treatment toxicity by selectively aiming at cells that express target receptors. Their efficacy depends on the level of receptors expressed on tumor cells as well as on the physicochemical properties of the tumor tissue that can hinder inratumor drug transport. To predict how to achieve maximal receptor saturation for a given tumor tissue we developed a procedure based on the microPKPD model simulations and data from dorsal window chamber (DWC) experiments [7,8]. The schematic of our approach is shown in Figure 2. Images from the DWC experiments (A) were digitized (B), and the tumor tissue architecture was explicitly reproduced in the mathematical model $(\mathrm{C})$. The process of ligand-receptor binding was quantified spatially (D), and the microPKPDsimulated drug transport (E) was used for calculations of the association kinetics for various ligand concentrations (F). Exploration of a model parameter space including diffusion, affinity, and release schemes $(\mathrm{G})$ led to scientific predictions (H). Using this approach, we tested which combinations of drug properties (diffusion, affinity), tissue topology (density, cellular loci), drug concentrations, and extravasation rates were critical for optimal drug delivery and desired cellular uptake on an individual cell level.

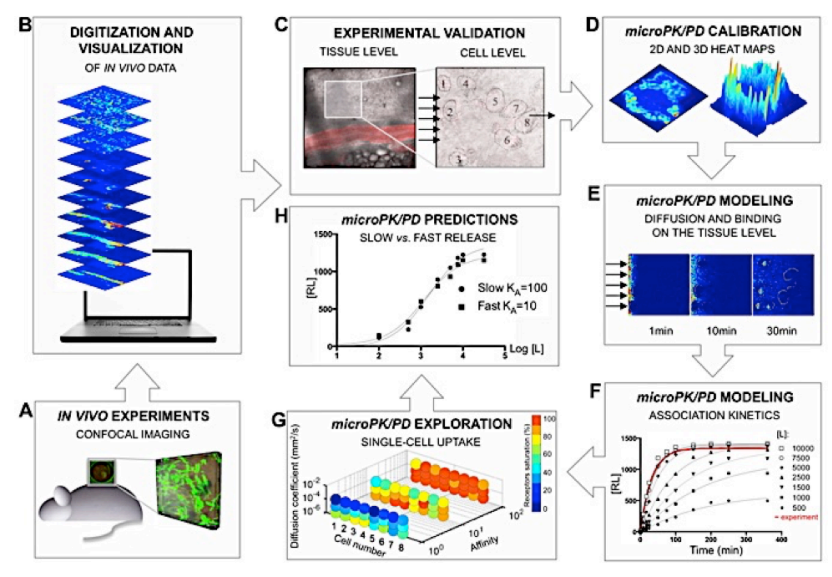

Figure 2. microPKPD applied to optimize targeted treatment properties and administration methods to maximize receptor saturation and uptake [7].

\section{Predicting chemotherapy response with Organoid3D}

Heterogeneity of tumor microenvironment $(\mathrm{mE})$, and the dynamic metabolic and structural changes that may occur in $\mathrm{mE}$ during the treatment may negatively influence efficiency of that therapy. To test interactions between $\mathrm{mE}$ and drugs, and to predict how tumors will respond to chemotherapy in various and variable microenvironments, we developed an in silico model of 3D tumor organoids, Organoid3D. The model can be calibrated to the properties of a specific cell line and a specific drug, and then used to simulate tumor spheroid growth in a given $\mathrm{mE}$ (hypoxia or normoxia, acidity or neutral $\mathrm{pH}$, high or low density of ECM, as well as gradients in all these features), and its response to various drugs and drug schedules. Figure 3 shows model interface, and results of MCF10A-1Ca spheroid growth simulations with no drug (left) and under a low level of doxorubicin (right) in a mild environment (normoxia, neutral $\mathrm{pH}$, low density ECM).



Figure 3. Interface of the in silico model Organoid $3 D$ for testing tumor response to chemotherapy in various and variable microenvironments [9].

\section{Quick Guide to Mathematical Methods}

The microPKPD models, including Organoid $3 D$, are based on the coupled reaction-diffusion equations of drug and nutrient kinetics $[7,8,10]$, and the particle-spring model for cell mechanics $[9,11]$.

\section{A. Equations}

Drugs are modeled either as discrete particles (Eq.1) that move by a random walk, a discrete diffusion process, and attach to cell membrane receptors according to binding kinetics (Eq.2); or as a continuous density (concentration) of molecules that move by diffusion and advection, and can be internalized by cells (Eq.3). Individual cells are subject to repulsive forces (Eq.4) that arise during cell division; they prevent cells from overlapping and collectively result in passive cell relocation (Eq.5). microPKPD is set up in the 2D space, while Organoid $3 D$ acts in 3D.

$$
\begin{aligned}
& (x, y)_{n+1}=\left\{\begin{array}{cc}
\left(X_{i}, Y_{i}\right)^{l} & \text { (a) if the binding condition is satisfied, } \\
(x, y)_{n} & \text { (b) if the particle crosses cell boundary, }
\end{array}\right. \\
& (x, y)_{n}+\sqrt{(2 D \Delta t)} \vartheta_{n} \quad \text { (c) otherwise, } \\
& B=B_{0}+\left(B_{\max }-B_{0}\right) /\left(1-e^{-k T}\right), \\
& \frac{\partial \eta_{a}(\mathbf{x}, t)}{\partial t}=\underbrace{\mathcal{D}_{\eta_{a}} \Delta \eta_{a}(\mathbf{x}, t)}_{\text {diffusion }}-\underbrace{\mathbf{u}(\mathbf{x}, t) \cdot \nabla \eta_{a}(\mathbf{x}, c)}_{\text {advection }}+\underbrace{\xi(c(\mathbf{x}, t)) \eta_{i}(\mathbf{x}, t)}_{\text {activation }}-\underbrace{\alpha \eta_{a}(\mathbf{x}, t) \chi\left(\Omega_{\mathrm{r}}\right)}_{\text {cellulur wptake }}, \\
& f_{i, j}=\left\{\begin{array}{ll}
\mathcal{F}\left(2 R_{C}-\left\|\mathbf{X}_{i}-\mathbf{X}_{j}\right\|\right) \frac{\mathbf{X}_{i}-\mathbf{X}_{j}}{\left\|\mathbf{X}_{i}-\mathbf{X}_{j}\right\|} & \text { if }\left\|\mathbf{X}_{i}-\mathbf{X}_{j}\right\|<2 R_{C} \\
0 & \text { otherwise, }
\end{array},\right. \\
& F_{i}=\Sigma_{j} f_{i, j}=-\nu \frac{d \mathbf{X}_{i}}{d t} \quad \text { and } \quad \mathbf{X}_{i}(t+\Delta t)=\mathbf{X}_{i}(t)-\frac{1}{\nu} \Delta t F_{i} .
\end{aligned}
$$

\section{B. Type of settings in which these methods are useful}

The microPKPD model can simulate drug transport through the tumor tissue based on either a patient's or mouse's histology, as well as drug action on a single-cell level. Organoid $3 D$ extends this model to $3 \mathrm{D}$ space. Both provide useful micropharmacology tools for optimizing treatment schedules, testing drug properties to improve their delivery and uptake, as well as predicting how a given tumor will respond to treatments of specific physicochemical properties and whether it will develop resistance. 


\section{REFERENCES}

[1] Laughney AM, Kim E, Sprachman MM, Miller MA, Kohler RH, Yang KS, Orth JD, Mitchison TJ, Weissleder R. Singlecell pharmacokinetic imaging reveals a therapeutic strategy to overcome drug resistance to the microtubule inhibitor eribulin. Science translational medicine 2014; 6(261):261 ra152.

[2] Vinegoni C, Dubach JM, Thurber GM, Miller MA, Mazitschek R, Weissleder R. Advances in measuring singlecell pharmacology in vivo. Drug Discov Today 2015; 20(9):1087-92.

[3] Dubach JM, Kim E, Yang K, Cuccarese M, Giedt RJ, Meimetis LG, Vinegoni C, Weissleder R. Quantitating drugtarget engagement in single cells in vitro and in vivo. Nat Chem Biol 2017; 13(2):168-73.

[4] Rejniak KA, Lloyd MC, Reed DR, Bui MM. Diagnostic assessment of osteosarcoma chemoresistance based on Virtual Clinical Trials. Med Hypotheses 2015; 85(3):348-354.

[5] Lloyd MC, Rejniak KA, Brown JS, Gatenby RA, Minor ES, Bui MM. Pathology to enhance precision medicine in oncology: lessons from landscape ecology. Adv Anat Pathol 2015; 22(4):267-272.

[6] Lloyd MC, Rejniak KA, Johnson JO, Gillies R, Gatenby R, Bui MM. Quantitative evaluation of the morphological heterogeneity in breast cancer progression. Modern Pathology 2012; 25:392A

[7] Karolak A, Rejniak KA. Mathematical modeling of tumor organoids: toward personalized medicine. 2017; in: Soker S, Skardal A, (Eds). Tumor Organoids: Springer.

[8] Karolak A, Estrella V, Chen T, Huynh A, Morse DL, Rejniak KA. Imaged-based computational predictions of imaging agent efficacy in pancreatic tumors expressing TLR2, Cancer Res. 2017; 77:A28;

[9] Karolak A, Huffstutler B, Markov DA, McCawley LJ, Rejniak KA. Elucidating the role of tumor microenvironmental heterogeneity with a computational model of $3 \mathrm{D}$ breast Spheroids, Biophysical Journal 2017; 112(3):45a

[10] Wojtkowiak JW, Cornell HC, Matsumoto S, Saito K, Takakusagi Y, Dutta P, Kim MJ, Zhang X, Leos R, Bailey KM, Martinez G, Lloyd MC, Weber C, Mitchell JB, Lynch RM, Baker AF, Gatenby RA, Rejniak KA, Hart C, Krishna MC, Gillies RJ. Pyruvate sensitizes pancreatic tumors to hypoxia-activated prodrug TH-302, Cancer \& Metabolism, 2015; 3:2

[11] Pérez-Velázquez J, Gevertz JL, Karolak A, Rejniak KA. Microenvironmental Niches and Sanctuaries: a Route to Acquired Resistance, Advances in Experimental Medicine and Biology, 2016; 936:149-164 